\title{
Fundamental Properties of a Large, Unbiased Sample of Massive, Young, Embedded Star Clusters in the Milky Way
}

\author{
Yigit Dallilar $^{1}$, Peter Barnes ${ }^{1,2}$, Elizabeth Lada ${ }^{1}$, Stuart Ryder ${ }^{3}$ \\ ${ }^{1}$ Astronomy Dept., University of Florida, Gainesville FL 32611, USA \\ email: ydallilar@ufl.edu, pjb@astro.ufl.edu \\ ${ }^{2}$ School of Science \& Technology, University of New England, Armidale NSW 2351, Australia \\ ${ }^{3}$ Australian Astronomical Observatory, PO Box 915, North Ryde NSW 1670, Australia
}

\begin{abstract}
In this project, we focus on the analysis of infrared observations of the clumps defined with the Galactic Census of High- and Medium-mass Protostars (ChaMP) (Barnes et al. 2011). We derive line of sight infrared extinction values, star counts and protostar candidates around the molecular gas emission obtained with the Mopra telescope. Then, we examine the correlation between radio and infrared properties of the clumps. For this stage of the project, we use the Vela-Carina and 2MASS catalogs to obtain a preliminary understanding of the final results. For the later stages, we will extract infrared photometry from our deep AAT near-IR and Spitzer 3.6 and $4.5 \mu \mathrm{m}$ images. With the final deep photometry results, we will compile the properties of individual clusters.
\end{abstract}

Keywords. Embedded clusters, Milky Way, extinction, star formation

\section{Introduction}

The Census of High- and Medium-mass Protostars (CHaMP) is the first large-scale, unbiased, uniform mapping survey of massive molecular clumps in the Milky Way (Barnes et al. 2011). Unlike other studies to investigate nearby cloud structures (such as Kong et al. 2015), this survey intends to describe a wide-field, unbiased overview of the massive young cluster distribution in a large portion of the Milky Way. This paper focuses on the cluster properties, such as the distribution of protostar candidates and line of sight extinction for each CHaMP clump. Here, we report our preliminary results from CHaMP fields and describe our future plans.

\section{Analysis \& Preliminary Results}

For the preliminary analysis, we exploited the Vela-Carina catalogs $\dagger$. Source catalogs for each field are obtained to cover the Mopra observations for the ChaMP clumps. For the first step, protostar candidates are identified for each field. We adopt Gutermuth et al.'s (2009) infared dereddening procedure. Color excess values from Flaherty et al. (2007) are taken as standard values. However, for $E_{J-H} / E_{H-K}$ color excess ratios, the 2MASS near-IR photometry catalog is used and we derived a near infrared extinction vector for each field. With this procedure, we can flag the sources which show a higher infrared excess than the color measurement errors in dereddened $[K-3.5]$ and $[3.5-4.6]$ colors.

$\dagger$ http://irsa.ipac.caltech.edu/data/SPITZER/GLIMPSE/catalogs/VELACAR/ 

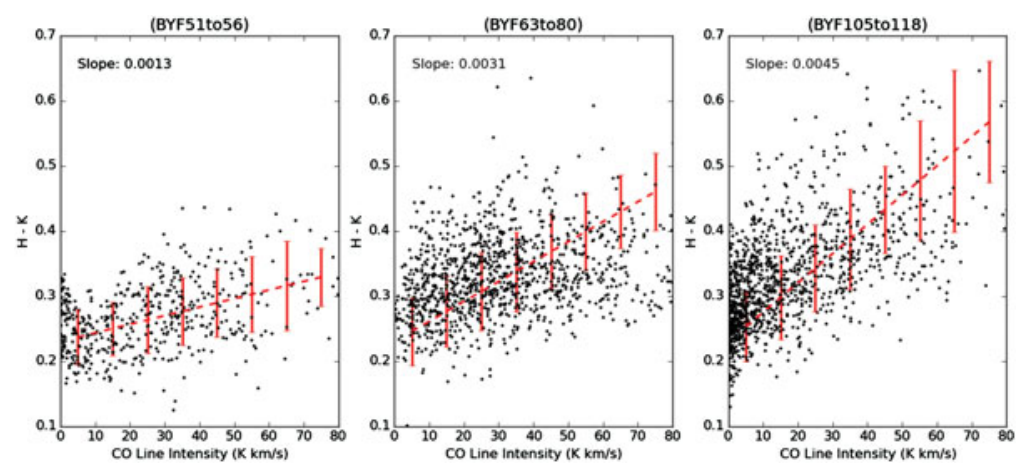

Figure 1. CO integrated line intensity versus $H-K$ color relation, and derived values for a substitute $X$-factor, for different CHaMP fields. From left to right, figures represents the fields BYF from 51 to 56,63 to 80,105 to 118 respectively, with slope values $0.0013,0.0031,0.0045$.

To obtain clean extinction maps, identified infrared excess sources or protostar candidates are excluded from these catalogs. $H-K$ colors for the rest of the objects are binned into the beam size of the Mopra observations. From these maps, we concluded that regions identified by high $\mathrm{CO}$ line intensity are correlated with relatively redder $H-K$ colors. Further, we looked for the pixel by pixel relation between CO total integrated line intensity and $H-K$ colors as a substitute for the column density/extinction relation. This allowed us to compute a linear relation for different fields, and we observed relatively different slopes for various regions. This suggests a clear change in the $X$-factor over different regions in the Carina Arm of the Milky Way (see figure 1 for three sample fields). If we assume a standard value from dust to gas ratio, $N\left(H_{2}\right) / A_{v}=9.4 \times 10^{20} \mathrm{~cm}^{-2} \mathrm{mag}^{-1}$ (Pineda et al. 2008), and $H-K$ color excess to extinction conversion, $A_{v} \sim 16 E(H-K)$, slope value of 0.0045 corresponds to $0.7 \times 10^{20} \mathrm{~cm}^{-2}(\mathrm{Kkm} / \mathrm{s})^{-1}$ for the $\mathrm{X}$ factor.

\section{Conclusion \& Future Plans}

We demonstrated our preliminary results on the extinction and protostar candidate counts for each CHaMP region exploiting the Vela-Carina catalogs. We derived $H-K$ color maps as a substitute for extinction and investigated its relation to the CO line intensity. Next, we will produce our own source photometry catalog for each region over our deep Spitzer images in the 3.5 and $4.6 \mu \mathrm{m}$ channels and AAT images for the near-IR bands. With this catalog, we will be able to track the extinction for each CHaMP clump instead of big fields. We will obtain the dust to gas ratio to characterise the evolutionary stage of each clump and constrain the clump sizes with a finer resolution on the extinction maps. For this purpose, we will measure the extinction law for each field separately and apply the NICEST method for extinction maps (Lombardi 2008). We intend to compare our results with studies on the nearby molecular cloud regions such as Kong et al. (2015).

\section{References}

Barnes, P. J., Yonekura, Y., \& Fukui, Y. et al. 2011, ApJ, 196, 12

Flaherty, K. M., Pipher, J. L., \& Megeath, S. L. et al. 2007, ApJ, 663, 1069

Gutermuth, R. A. \& Megeath, S. T., Myers et al. 2009, ApJ, 184, 22

Kong, S., Lada, C. J., \& Lada, E. A. et al. 2015, A\&A, 805, 19

Lombardi, M. 2008, A\&SA, 493, 735

Pineda, J. E., Caselli, P., \& Goodman, A. A. 2008, ApJ, 679, 481 